\title{
Enhanced numerical design of two-barrier infrared detectors with III-V compounds heterostructures considering the influence of lattice strain and misfit dislocations on the band gap
}

\author{
A. Jóźwikowska ${ }^{1} \cdot$ M. Suligowski ${ }^{2} \cdot$ K. Jóźwikowski ${ }^{2}$
}

Received: 30 November 2018 / Accepted: 3 July 2019 / Published online: 10 July 2019

(C) The Author(s) 2019

\begin{abstract}
The goal of this work is the design of efficient infrared radiation detectors based on InAsSb compounds with two energy barriers around the absorber region. Two types of two-barrier detectors that work in the 3-5.5 $\mu \mathrm{m}$ wavelength range at $230 \mathrm{~K}$ is designed. Using our computer program to iteratively solve the Poisson equation, the spatial distribution of energy band edges in III-V heterostructures was calculated The influence of lattice stress, bending of the structure and doping on the energy shift of the edge of the bands is considered. Lattice strain is the cause of the formation of the misfit dislocations at the boundaries of the individual layers of the heterostructure. These dislocations partially relax the resulting stress and affect the lattice deformation in individual layers. From the minimum elastic energy condition, the density of these dislocations is determined. It has been shown that the band offset can be eliminated in the areas of both barriers in the designed two-barrier detectors.
\end{abstract}

Keywords SUBCOOLED boiling · CFD · Two-fluid model $\cdot$ Interfacial heat transfer

\section{Introduction}

In recent years, we have been observing the development of theoretical and technological research on heterostructures from InAsSb intended for the construction of infrared detectors - see for example, works (Klipstein et al. 2012; Craig et al. 2015, 2016; Ting et al. 2016;

This article is part of the Topical Collection on Numerical Simulation of Optoelectronic Devices, NUSOD' 18.

Guest edited by Paolo Bardella, Weida Hu, Slawomir Sujecki, Stefan Schulz, Silvano Donati, Angela Traenhardt.

K. Jóźwikowski

krzysztof.jozwikowski@wat.edu.pl

1 Faculty of Applied Informatics and Mathematics, Warsaw University of Life Science SGGW, Nowoursynowska 166 St., 02-787 Warsaw, Poland

2 Institute of Applied Physics, Military University of Technology, 2 Gen. Urbanowicza St., 00-908 Warsaw, Poland 
Gomólka et al. 2017). Of interest are $\operatorname{InAs}_{0.75} \mathrm{Sb}_{0.25}$ structures used for the construction of uncooled or thermoelectrically cooled infrared detectors in the range of 3-5.5 $\mu \mathrm{m}$ (middle wavelength infrared radiation (MWIR)). These detectors are manufactured for example, by Hamamatsu, and Vigo System SAcompanies. Despite the fact that there are a few percent misfit of lattice constants between the InAsSb layer and GaAs or GaSb, the latter two materials are most often used as substrates. The resulting lattice stress induces significant lattice strain in the heterostructures. To design infrared detectors based on the above mentioned heterostructures, we must consider the effect of these deformations on the physical properties of the individual layers. The situation is further complicated by the deposition of additional layers acting as energy barriers for electrons or holes (Klipstein et al. 2012). Strain causes a fractional volume change that implies the change in the width of the energy gap. In addition, shear strain splits and lines up the valence bands and the indirect conduction bands. Although the problem is important for technologists and designers of infrared detectors, it is difficult to find a recent work in which it would be analyzed. The problem of lattice strain was, however, analyzed in the case of a superlattice (Smith and Mailhiot 1987; Plis et al. 2010). Assumption of a pseudomorphic interface enables for a simple determination of strain in the individual layers of the heterostructure. Usually, however, the deformations are much smaller than anticipated. The reason for that are misfit dislocations generated in the areas of interfaces. This problem is described in detail in our previous work devoted to InAsSb heterostructures deposited on a GaAs substrate (Jóźwikowska et al. 2019). In that paper we obtained expressions on the shift of the edge of the conduction band and of the valence band caused by doping, the lattice strain and the bending of structures. Additionally, from the minimum elastic energy condition, we determined the density of misfit dislocations in individual interfaces of the heterostructures. We verified our calculations by comparing them with experimental results obtained by (Kim and Razeghi 1998; Xie et al. 2018). In this work, we use the same methods as in our work (Jóźwikowska et al. 2019). We refer to that publication for their detailed descriptions.

The aim of this work is to design two-barrier detectors from InAsSb heterostructures, taking into account the influence of the lattice strain and misfit dislocations on their band structure. We are not aware of any papers in which this problem would be considered.

The key to designing two-barrier detectors is to determine, (in thermal equilibrium conditions), the spatial distribution of the apex of the valence band and the valley of the conduction band. In III-V compounds, the character of the energy gap may change, depending on the molar composition and temperature. Therefore, the minimum of the conduction band with the points $\Gamma, \mathrm{X}$ and $\mathrm{L}$ of the Brilouine zone should be considered (Vurgaftman et al. 2001). In turn, to correctly calculate the spatial distribution of the electrostatic potential, which has a fundamental influence on the spatial distribution of the energy band edges, we must consider the concentration of various types of carriers occupying different energy bands. We calculate the electrostatic potential by solving numerically the Poisson equation, using an iterative method (Jóźwikowska 2008).

Heterostructures designed in this paper consist of a substrate made from a weakly doped $\mathrm{GaSb}$ or GaAs. As the buffer layer, we used a heavily doped n type InAs. The weakly doped $\mathrm{p}$ type $\mathrm{InAs}_{0.25} \mathrm{Sb}_{0.75}$ absorber is surrounded by thin AlAsSb layers, separating the absorber from the buffer layer and the heavily doped thin layer of the p type InAs cap layer. AlAsSb layers act as energy barriers for electrons or holes depending on the type of dopants they contain. They should be strongly doped.

Then, polarizing structures in the reverse direction (similarly as it happens for structures with HgCdTe (Jóźwikowski and Jóźwikowska 2019), we can cause the effect of exclusion and extraction of charge carriersin the absorber area, which will reduce the concentration 
of carriers in the absorber area (Ashley and Elliott 1985; White 1988). As a result, the carrier generation rate and the recombination rate are suppressed, the generation-recombination (G-R) noise is reduced and the quantum efficiency and detectivity are increased.

A two-barrier detector working in a non-equilibrium mode of operation (NEMO) can be an alternative to structures with an absorber made of a superlattice of type II. The superlattices are used to limit the Auger G-R processes. There is a small mobility of electrons in superlattices, which may be an obstacle to obtaining high-performance detectors. In addition, two-barrier detectors are much easier to grow than the superlattices.

The main problem, in the case of barrier detectors, is the occurring band offset in the areas where the absorber meets the barrier areas. Its effective elimination is the key to making high-performance detectors. Band offset is limited by the appropriate selection of gap width and doping of barriers and areas surrounding the absorber.

Designing heterostructures with an appropriate band structure is the purpose of this work.

\section{Numerical method and results of calculations}

In this work we analyze two uncooled cylindrical mesa two-barrier detectors, (Fig. 1) with the surface area of $250 \mu \mathrm{m}^{2}$. The $\operatorname{InAs}_{1-\mathrm{x}} \mathrm{Sb}_{\mathrm{x}}$ epitaxial layer is grown on a $300 \mu \mathrm{m}$-thick $\mathrm{GaSb}$ (structure A), or GaAs substrate (structure B). Spatial distribution of mole fraction and dopant concentrations in epitaxial layers are shown in Fig. 2. Figure 2 and all the remaining figures present the distributions of parameters of these heterostructures and selected physical quantities as functions of thickness along the axis of symmetry shown in Fig. 1.

Heterostructures from $\mathrm{HgCdTe}$ have the lattice constants very well-matched. The difference between the lattice constant of HgTe and CdTe is only $1.5 \times 10^{-2} \AA$ (Capper and Garland 2011). Therefore, when designing $\mathrm{HgCdTe}$ heterostructures, one usually neglects lattice stress, which is small. When we look at Figs. 3 which show the values of the lattice constant in the A and B structures, we notice that the differences in lattice constants

Fig. 1 The architecture of the half cross-section of cylindrical $\mathrm{P}^{+} \mathrm{nN}^{+}$MWIR mesa structure with two barriers. Structure A is grown on $\mathrm{GaSb}$, structure $\mathrm{B}$ is grown on $\mathrm{GaAs}$ substrate

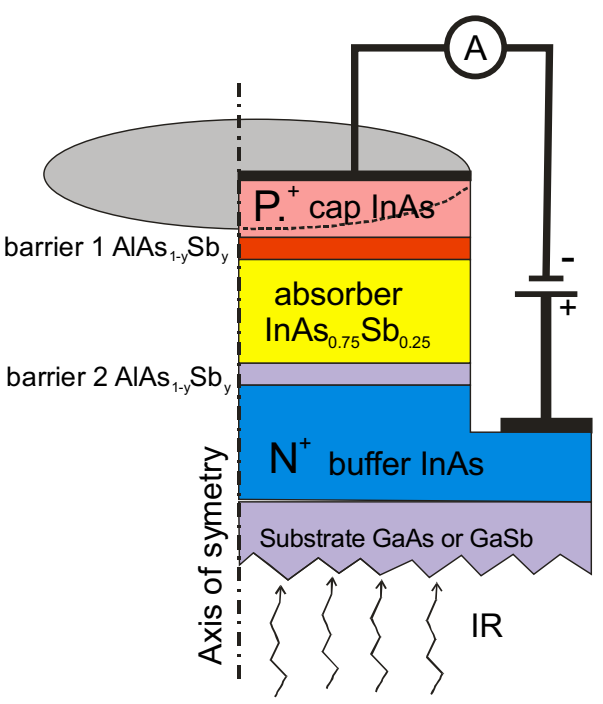



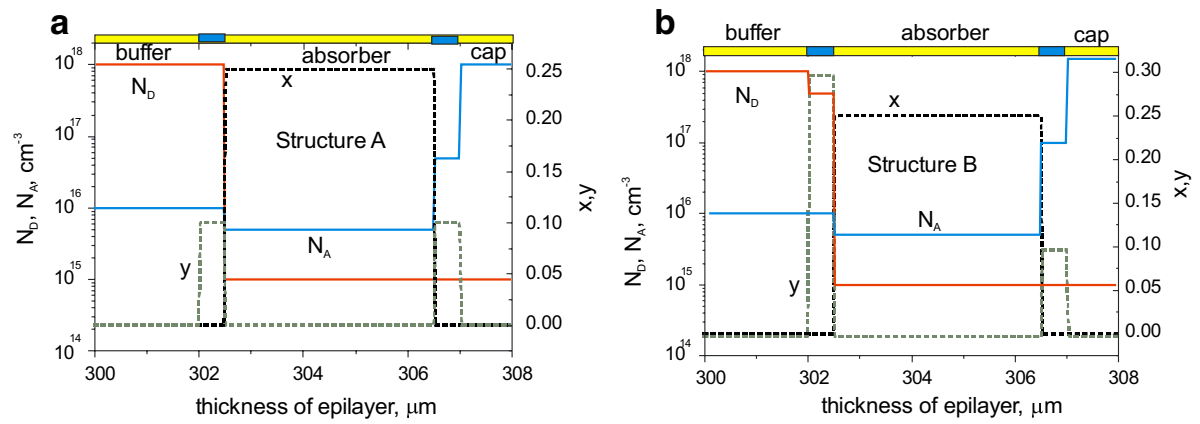

Fig. 2 Spatial distribution of the mole fraction $\mathrm{x}$ (for a $\mathrm{InAs}_{1-\mathrm{x}} \mathrm{Sb}_{\mathrm{x}}$ heterostructure), the mole fraction $\mathrm{y}$ (for barriers with $\mathrm{AlAs}_{1-\mathrm{y}} \mathrm{Sb}_{\mathrm{y}}$ ), concentration of donors $N_{D}$ and acceptors $N_{A}$ in epilayers working at $230 \mathrm{~K}$. a Concerns structure A, b Concerns structure B
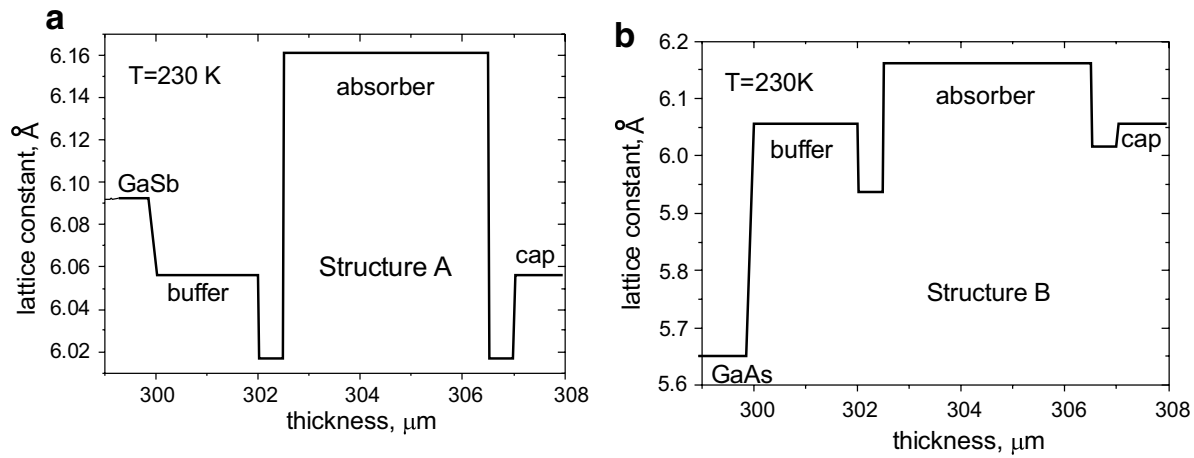

Fig. 3 Spatial distribution of a lattice constant in heterostructures A (a) and B (b)

Fig. 4 A diagram of a mesa structure with a cylindrical symmetry. Forces marked as $F_{B}$ are internal forces due to lattice mismatches between the individual layers of the hetrostructure

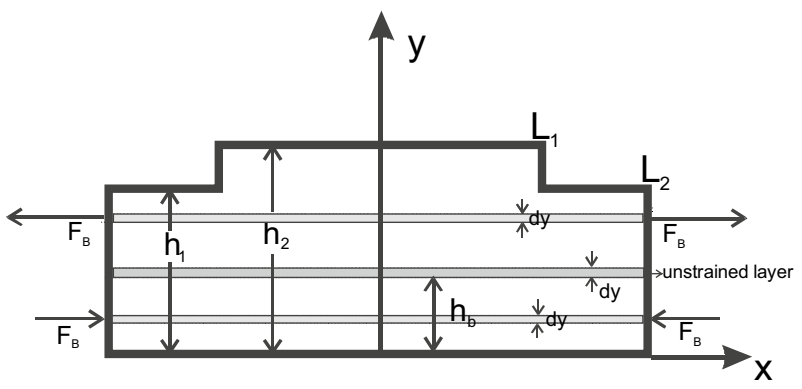

of individual layers are at least an order of magnitude higher than for the heterostructures with $\mathrm{HgCdTe}$. This is the reason for the occurrence of large lattice stress causing large lattice strain. Internal forces shown in Fig. 4 cause such deformations of the lattice in the planes of growth of the heterostructures (marked as xz) that the lattice constant in all the heterostructure is the same and is equal $a_{\|}$. In (Jóźwikowska et al. 2019), we derived the formula for $a_{\|}$in the mesa cylindrical structures. For the structure in Fig. 4, it equals: 


$$
\begin{gathered}
a_{\|}=\frac{L_{1}^{2} \int_{h_{1}}^{h_{2}} \frac{G(y)}{a_{0}(y)} d y+L_{2}^{2} \int_{0}^{h_{1}} \frac{G(y)}{a_{0}(y)} d y}{L_{1}^{2} \int_{h_{1}}^{h_{2}} \frac{G(y)}{a_{0}^{2}(y)} d y+L_{2}^{2} \int_{0}^{h_{1}} \frac{G(y)}{a_{0}^{2}(y)} d y} \\
\text { where } G(y)=2\left(c_{11}(y)+2 c_{12}(y)\right)(1-D(y) / 2) .
\end{gathered}
$$

$D$ depends on the elasticity constants $c_{11}, c_{12}$ and $c_{44}$ of the given material and the orientation of the interface plane, and so

$$
D^{001}=2 \frac{c_{12}}{c_{11}} \quad D^{110}=\frac{c_{11}+3 c_{12}-2 c_{44}}{c_{11}+c_{12}+2 c_{44}} \quad D^{111}=2 \frac{c_{11}+2 c_{12}-2 c_{44}}{c_{11}+2 c_{12}+4 c_{44}}
$$

The structure shown in Fig. 4 is not in static equilibrium despite the balance of internal forces. Only the bending enables achieving the balance of momenta of the internal forces due to the appearance of an additional stress, which partially changes the stress responsible for the formation of a pseudomorphic structure (constant $a_{\|}$). Some of the layers are subject to an additional tensile and some to an additional compression. Bending of the structure enables for example such situations as shown in Fig. 5, where lower layers are compressed, and the upper ones are tensed. The total sum of the vertical components of forces $d F_{y}=d F \sin \phi_{2}$ in the base of the mesa and $d F_{y}=d F \sin \phi_{1}$ in the upper part should also be zero under equilibrium conditions. The parameters to be determined are the radius of curvature $R$ and $h_{b}$ the distance of the undeformed layer from the base of the heterostructure. These quantities were derived in our earlier paper (Jóźwikowska et al. 2019):

$$
R \approx \frac{L_{2}^{2} \int_{0}^{h_{1}} y G(y) \frac{a_{\|}}{a(y)} d y+L_{1}^{2} \int_{h_{1}}^{h_{2}} y G(y) \frac{a_{\|}}{a(y)} d y}{L_{2}^{2} \int_{0}^{h_{1}} G(y)\left(1-\frac{a_{\|}}{a(y)}\right) d y+L_{1}^{2} \int_{h_{1}}^{h_{2}} G(y)\left(1-\frac{a_{\|}}{a(y)}\right) d y} .
$$

and

$$
h_{b}=\frac{L_{2} \int_{0}^{h_{1}} G(y)(R+y)^{2} \frac{a_{\|}}{a(y)} d y+L_{1} \int_{h_{1}}^{h_{2}} G(y)(R+y)^{2} \frac{a_{\|}}{a(y)} d y}{L_{2} \int_{0}^{h_{1}} G(y)(R+y) d y+L_{1} \int_{h_{1}}^{h_{2}} G(y)(R+y) d y}-R
$$

Fig. 5 An example of an internal force acting on a selected element of the structure after bending. The resulting moment of all internal forces acting on the heterostructure when equilibrium is reached equals zero

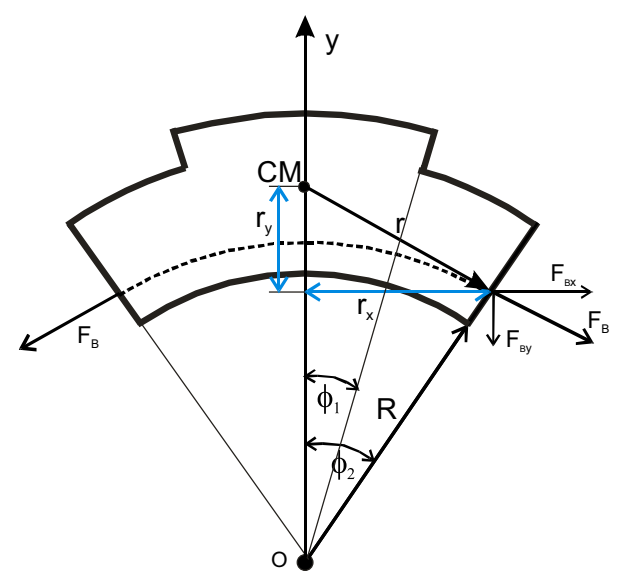


Fig. 6 To partially relax biaxial stress, dislocations created form a matrix with equally spaced dislocation lines which are mutually perpendicular to each other

Fig. 7 A diagram of the analyzed structures. The Volterra construction allows analyzing dislocations marked $\Delta$ as if the entire lattice plane (marked with a dashed line) has been removed from the heterostructure. Those marked as $\nabla$ are analyzed as if we added the entire lattice plane (marked with a solid line). These dislocations differ by the signs of the Burgers vector
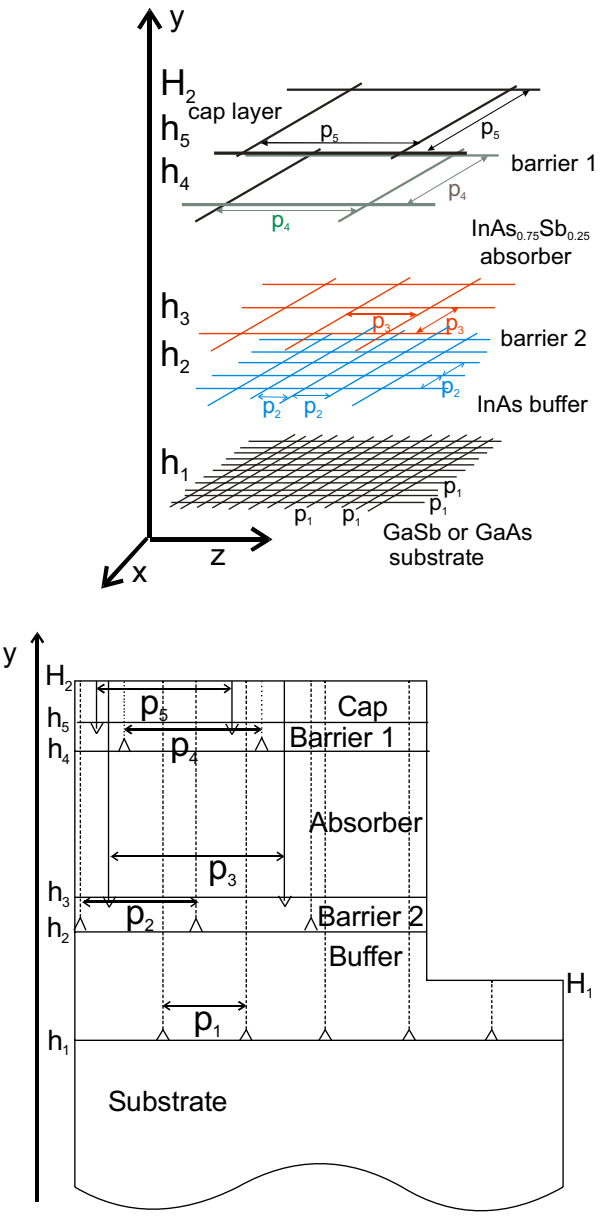

where $a(y)$ denotes the lattice constant in the structure without stress.

It is strongly documented experimentally, that to partially relax biaxial stress, dislocations created form a matrix with equally spaced dislocation lines which are mutually perpendicular to each other. Their schematic diagram for the heterostructure analyzed in the paper is shown in Fig. 6. Following (Gosling et al. 1993), we adapted their method for a multi-layer structure consisting of several layers (differing by the lattice constants) and interfaces being the places of the generation of misfit dislocations. Figure 7 shows the diagram of the photodiode analyzed by us. We described it in detail in (Jóźwikowska et al. 2019).

The elastic energy per unit area, $\breve{E}$, of the heterostructure is expressed by the dependence [after (Gosling et al. 1993; Jóźwikowska et al. 2019):

$$
\begin{aligned}
\check{E}= & \frac{1}{2} \int_{0}^{h} B\left(\frac{a_{\|}-a}{a}\right)^{2} d y+\frac{1}{2} \sum_{i=1}^{5}\left(\frac{b_{1}\left(h_{i}\right)}{p\left(h_{i}\right)}\right)^{2} \int_{h_{i}}^{H_{2}} B d y+\sum_{i=1}^{5} \frac{b_{1}\left(h_{i}\right)}{p\left(h_{i}\right)} \int_{h_{i}}^{H_{2}} B\left(\frac{a_{\|}-a}{a}\right) d y \\
& +\sum_{j=1}^{4} \frac{b_{1}\left(h_{j}\right)}{p\left(h_{J}\right)}\left(\sum_{i=j+1}^{5}\left(\frac{b_{1}\left(h_{i}\right)}{p\left(h_{i}\right)}\right)^{2} \int_{h_{i}}^{H_{2}} B d y\right)
\end{aligned}
$$


To calculate the minimum of the energy $\breve{E}$ we numerically determined the values of $p_{1}, p_{2}, p_{3}, p_{4}$ and $p_{5}$. If the epitaxial layer contains dislocations with the average inter-dislocation p, the expression for strain becomes (Jain et al. 1992, 1993, 1997; Gosling et al. 1993; Jóźwikowska et al. 2019)

$$
\varepsilon_{\|}(y)=\frac{a_{\|}-a(y)}{a(y)}+\frac{y-h_{b}}{R}-\sum_{i=1}^{k} b_{1}\left(h_{i}\right) / p\left(h_{i}\right)
$$

where $b_{1}=-b \sin \alpha \sin \beta$ and $b$ is the magnitude of the Burgers vector, $h_{i}$ is the y coordinate of the i-th interface, $k$ is selected so that $h_{k}<y . a(y)$ is the lattice constant in the layer without stress. The above values of $b_{1}$ are valid for dislocations marked as $\Delta$ (Fig. 7), while for dislocations marked as $\nabla$, b1 has an opposite sign (Jain et al. 1992, 1993). For $60^{\circ}$ dislocations $\alpha=\operatorname{arctg} \frac{1}{\sqrt{2}}, \beta=\frac{\pi}{3}$.

Generally, dislocations reduce the lattice strain. However, it is possible that in some areas of the heterostructure they increase the strain or change its character from tensile to compressive or vice versa, in such a way that the minimum elastic energy in the whole structure is reached.

Figure 8a shows the spatial distribution of the lattice strain in the GaSb substrate (structure A) with a thickness of $300 \mu \mathrm{m}$. Figure $8 \mathrm{~b}$ describes the GaAs substrate of the same thickness (structure B). The layers were deposited on these substrates whose spatial distribution of the molar composition and doping is depicted in Fig. 2a, b, respectively. The figures also show the values of the parameters $\mathrm{R}$ and $h_{b}$. Much greater lattice misfit between GaAs and the epilayer than for GaSb is the cause of higher bending and the strain more than an order of magnitude bigger in GaAs. Figure 9 show the spatial distribution of strain in epitaxially deposited structures A and B. In the absorber layer, both in structure A and $\mathrm{B}$, strain is compressive and amounts to about $0.75-0.8 \%$. This causes an increase in the energy gap around $0.05 \mathrm{eV}$ Generated dislocations in structure A reduce strain in the absorber area, and in other areas they cause its slight increase. In the entire epitaxial layer of structure B, strain is compressive, and dislocations everywhere reduce these deformations. However, both in the region of the two barriers and the cap layer, the dislocations cause changes in the kind of a strain.
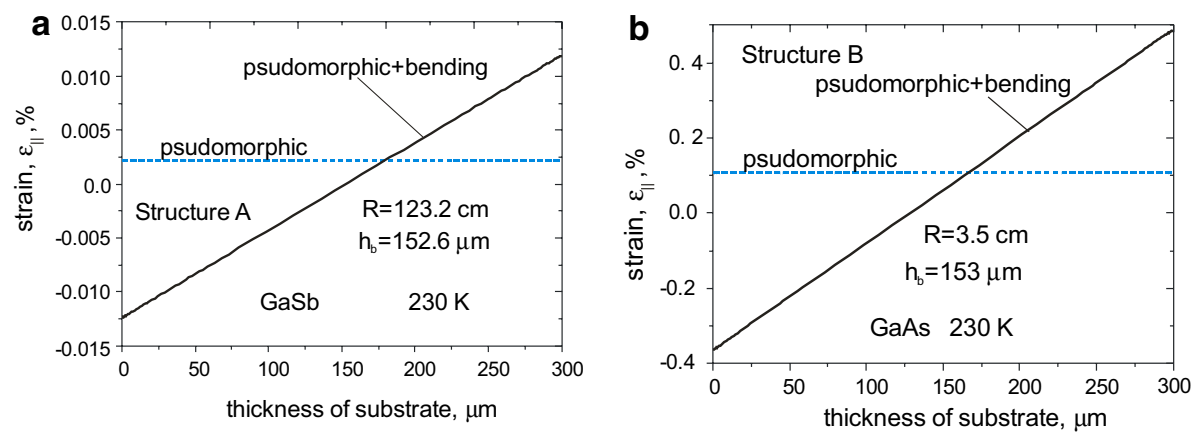

Fig. 8 Spatial distribution of lattice strain in the substrate of structure A (a) and B (b). The dashed line indicates the pseudomorphic strain, the continuous line is the strain created when we additionally consider the bending of the structure 

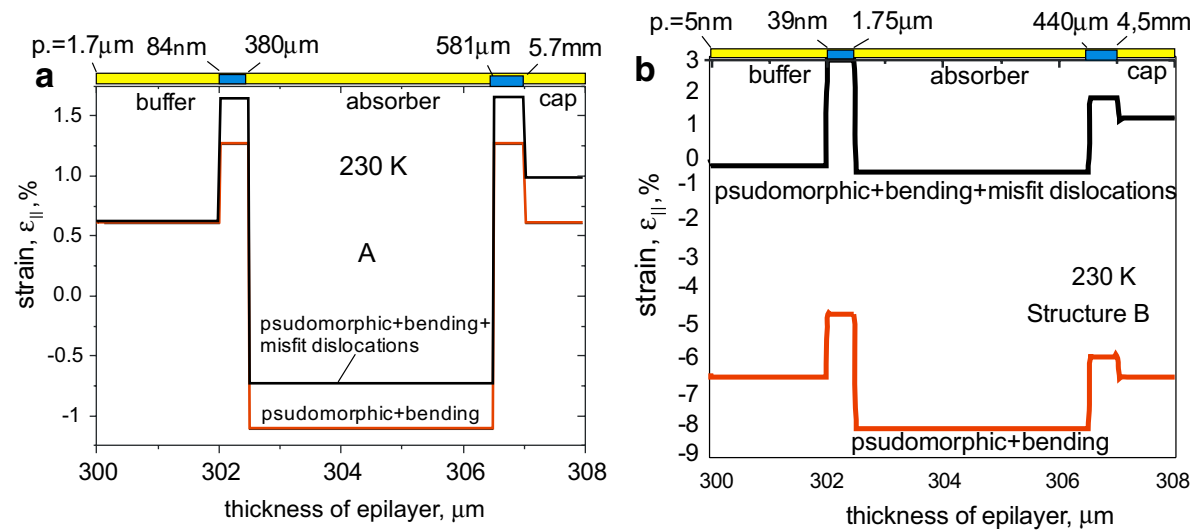

Fig. 9 The spatial distribution of the lattice strain in the epitaxial layer of the heterostructure A (a) and B (b). The red lines show strain caused by pseudomorphic lattice misfit and bending, the black lines show strain partly relaxed by misfit dislocations. The numbers at the heterostructures' interfaces represent the distances between lines of misfit dislocations in interfaces' areas

Figure 10 show the average lattice strain in the absorber area as a function of thickness of the InAs buffer for three selected substrate thicknesses. The relationship shows an oscillatory character. In the structure A, along with the thickness of the buffer, the compressive strain increases in the absorber area. In structure $\mathrm{B}$, compressive strain increases with increasing the buffer thickness to $8 \mu \mathrm{m}$, and then it decreases. For structures with thinner substrates, it is possible to change the nature of strain to tensile strain. Figure 11 presents the spatial distribution of lattice strain in the substrate of a structure with a thickness of $200 \mu \mathrm{m}$. The bending radius is only $9 \mathrm{~mm}$ and the height $\mathrm{h}_{\mathrm{b}}=107 \mu \mathrm{m}$. The bending of the structure is very strong, the compressive strain in the lower part of the substrate and the tensile strain in the upper part are of the order of $1 \%$.

A layer with a $10 \mu \mathrm{m}$ thick buffer was deposited on this substrate. Figure 12 shows the spatial distribution of the deformations in this layer. The resulting stress generates misfit dislocations of high density in the interface of the buffer-substrate and the
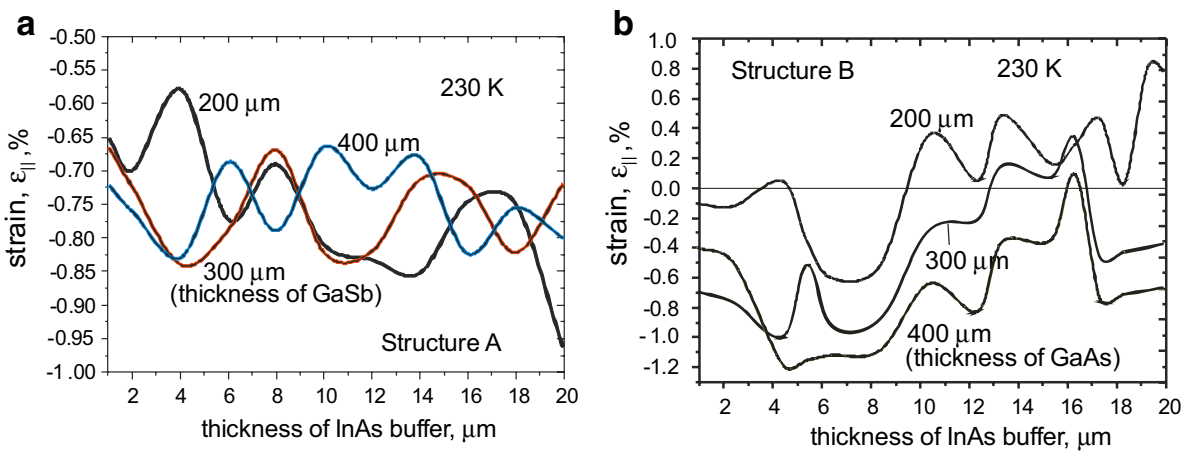

Fig. 10 Average lattice strain in the absorber area as a function of thickness of the InAs buffer for three selected substrate thicknesses: GaSb (a) and GaAs (b) 
Fig. 11 Spatial distribution of lattice strain in the substrate with the thickness of $200 \mu \mathrm{m}$ for structure B The dashed line denotes the pseudomorphic strain, the solid line the strain created when we additionally consider the bending of the structure

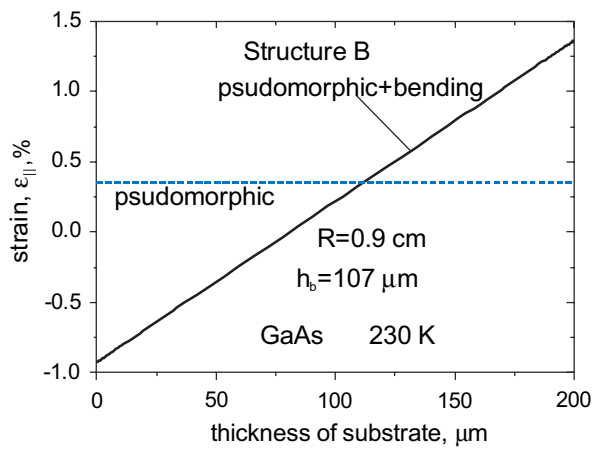

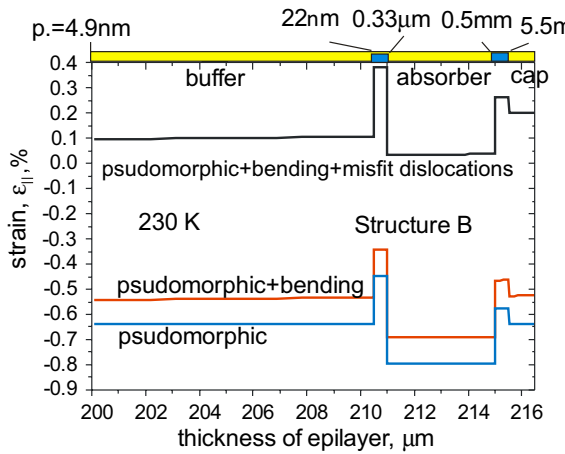

Fig. 12 The spatial distribution of the lattice strain in the epitaxial layer of the heterostructure B. The blue line shows deformations caused by pseudomorphic lattice misfit. The red line is the strain created when we additionally. consider the bending of the structure, the black line shows strain partly relaxed by misfit dislocations. The numbers at the heterostructures interfaces represent the distances between misfit lines in the interfaces' areas. (Color figure online)

buffer-barrier 2. Dislocations reduce stress and cause changes in the character of strain. In the absorber area, they now have very small values below $0.5 \%$.

The lattice strain caused by the lattice misfit has a strong impact on the energy bands' shifting. Hydrostatic strain leads to the band shifts expressed by relation:

$$
\Delta E_{V, a v}=a_{V}\left(\epsilon_{\perp}+2 \epsilon_{\|}\right) \quad \Delta E_{C}=a_{C}\left(\epsilon_{\perp}+2 \epsilon_{\|}\right)
$$

Here $E_{V, a v}$ is the average of three uppermost valence bands at $\Gamma$ introduced by (Van de Walle 1989). $E_{C}$ is the edge of conduction band.

Direct conduction bands (at $\Gamma$ ) are nondegenerate and therefore are only subject to hydrostatic strain shift. Shear components of the strain can have a profound effect on degenerate bands. They lead to splitting of the valence bands (and of indirect conduction bands) which are well-described by a deformation potential theory and were discussed by (Van de Walle 1989). The strain splittings themselves are proportional to the magnitude of the strain and are well-described in terms of deformation potentials. For strain along [001], the following shifts are calculated with respect to the $E_{V, a v}$ (as in Van de Walle 1989)

$$
\Delta E_{h h}=\frac{1}{3} \Delta_{o}-\frac{1}{2} \delta_{001}
$$




$$
\begin{aligned}
& \Delta E_{l h}=-\frac{1}{6} \Delta_{o}+\frac{1}{4} \delta_{001}+\frac{1}{2}\left[\Delta_{0}^{2}+\Delta_{o} \delta_{001}+\frac{9}{4}\left(\delta_{001}\right)^{2}\right]^{1 / 2} \\
& \Delta E_{S O}=-\frac{1}{6} \Delta_{o}+\frac{1}{4} \delta_{001}-\frac{1}{2}\left[\Delta_{0}^{2}+\Delta_{o} \delta_{001}+\frac{9}{4}\left(\delta_{001}\right)^{2}\right]^{1 / 2}
\end{aligned}
$$

In these equations $\delta_{001}$ is given by:

$$
\delta_{001}=2 k\left(\epsilon_{\perp}-\epsilon_{\|}\right)
$$

Here $k$ is the shear deformation potential and $\Delta_{o}$ is the spin-orbit splitting. In semiconductors where the band gap is indirect (in AlAsSb) we need, however, to analyze the strain splitting of the indirect conduction-band minima. The conduction band minimum at $\mathrm{X}$ are not affected by uniaxial strain along the [111] direction. Under uniaxial strain along [001] or [110] the bands along [100] and [010] split from the ones along [001]. The splitting of the bands with respect to the direction is then given by:

$$
\Delta E_{C}^{001}=\frac{2}{3} b\left(\epsilon_{\perp}+2 \epsilon_{\|}\right) \quad \Delta E_{C}^{100,010}=-\frac{1}{3} b\left(\epsilon_{\perp}+2 \epsilon_{\|}\right)
$$

Because there are six equivalents $0,0,1$ directions, the conduction-band valleys all coincide in the equilibrium but can be split by the application of strain in appropriate directions. In that case as shown by (Van de Walle 1989) the value $E_{C}$ should be considered as an average over the conduction-band valleys in spatially distinct directions. This average is shifted due to hydrostatic strains, using the values $a_{C}$ for indirect energy gap listed in (Van de Walle 1989). All parameters for the ternary compounds analyzed in this work depend on their molar compositions according to the relationships given in the work (Vurgaftman et al. 2001), or the references therein.

Figure 13 shows the influence of strain $\varepsilon_{\|}$on the position of the band edges in the absorber. Calculations made by us based on the relations obtained in (Van de Walle 1989; Jóźwikowska et al. 2019). As one would expect, compression strain increases the width of the energy gap and stretch decreases it. In this work, we considered the influence of lattice strain on the position of the edges of the bands in the whole heterostructure. In addition to lattice deformation, also the electric potential affects their position. To determine the electric potential distribution, we must solve the Poisson equation.

The calculation procedures for $E_{V, a v}$ for ternary compounds are presented in (Van de Walle 1989).

Fig. 13 Effect of strain $\varepsilon_{\|}$on the position of the edge of conduction band, $\mathrm{E}_{\mathrm{C}}$, the averaged valence band edge, $\mathrm{E}_{\mathrm{V}, \mathrm{av}}$, the heavy hole band edge, $\mathrm{E}_{\mathrm{hh}}$, the light hole band edge, $\mathrm{E}_{\mathrm{lh}}$ and the edge of the split-off spin-orbit band, $\mathrm{E}_{\mathrm{SO}}$ in $\operatorname{InAs}_{0.25} \mathrm{Sb}_{0.75}$ structures

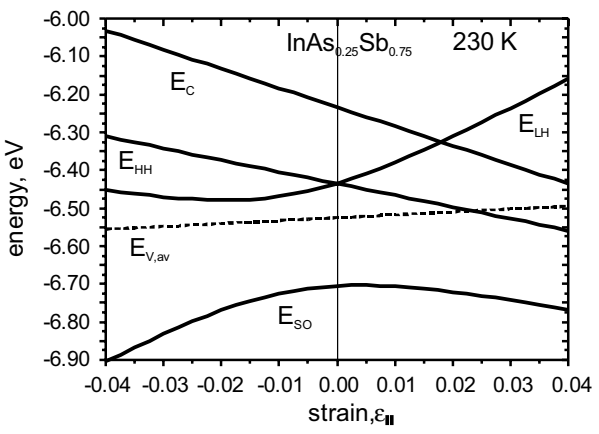


Position of the topmost valence band reads:

$$
E_{V}=E_{V, a v}+\Delta E_{V, a v}+\frac{\Delta_{0}}{3}-e \Psi
$$

where $\Delta_{0}$ is the energy of spin-orbit splitting, $e$ is the elementary charge and $\Psi$ is the electrostatic potential. Considering the values of conduction band minima, we can determine the lowest energy gap $E_{g}$, then:

$$
E_{C}=E_{V}+E_{g}+\Delta E_{C}-e \Psi
$$

is the value of minimum of conduction band.

Next we solve the nonlinear Poisson equation (Jóźwikowska 2008) to obtain the spatial distribution of electrical potential and, eventually, the distribution of the edge of conduction and valence bands

$$
\nabla^{2} \Psi=-\frac{e}{\varepsilon \varepsilon_{0}}\left(N_{D}^{+}-N_{A}^{-}+p_{h h}+p_{l h}-n_{\Gamma}-n_{X}-n_{L}\right)-\frac{1}{\varepsilon} \nabla \Psi \nabla \varepsilon
$$

Boundary conditions for the Poisson Eq. (14) are assumed to be the Neuman conditions i.e.: $\nabla \Psi=0$. where $N_{D}^{+}, N_{A}^{-}, p_{l h}, p_{h h}, n_{\Gamma}, n_{X} a n d n_{\mathrm{L}}$, are the concentration of ionized donors, ionized acceptors, holes in the light hole band, holes in the heavy hole band, electron concentration in the valley $\Gamma$, in the $X$ valleys, and in the L valleys, respectively. $\varepsilon$ is the static dielectric permittivity (Adachi 2005; Vurgaftman et al. 2001), $\varepsilon_{0}$ the dielectric permittivity of the vacuum, and $\Psi$ denotes the electrostatic potential. Concentrations of carriers and ionized dopants are calculated from standard dependencies (e.g. Blakemore 1962; Look 1989; Singh 1993):

$$
\begin{gathered}
n_{\Gamma}=\int_{B Z} \frac{1}{\pi} k^{2} d k\left[1+\exp \left(\frac{E_{C}^{\Gamma}-F}{k_{B} T}\right)\right]^{-1} \\
n_{X}=\int_{0}^{\infty} 24 \pi\left(\frac{2}{h^{2}}\right)^{\frac{3}{2}}\left[m_{l}^{*}\left(m_{t}^{*}\right)^{2}\right] E_{k}^{1 / 2} d E_{k}\left[1+\exp \left(\frac{E_{C}^{X}-F}{k_{B} T}\right)\right]^{-1} \\
n_{L}=\int_{0}^{\infty} 16 \pi\left(\frac{2}{h^{2}}\right)^{\frac{3}{2}}\left[m_{l}^{*}\left(m_{t}^{*}\right)^{2}\right] E_{k}^{1 / 2} d E_{k}\left[1+\exp \left(\frac{E_{C}^{L}-F}{k_{B} T}\right)\right]^{-1} \\
p_{h h}=\int_{B Z} \frac{1}{\pi} k^{2} d k\left[1+\exp \left(\frac{F-E_{h h}}{k_{B} T}\right)\right]^{-1} \\
p_{l h}=\int_{B Z} \frac{1}{\pi} k^{2} d k\left[1+\exp \left(\frac{F-E_{l h}}{k_{B} T}\right)\right]^{-1}
\end{gathered}
$$




$$
\begin{aligned}
& N_{A}^{-}=\frac{N_{A}}{1+2 \exp \left(\frac{E_{V}+E_{A}-F}{k_{B} T}\right)} \\
& N_{D}^{+}=\frac{N_{D}}{1+4 \exp \left(\frac{F-E_{C}-E_{D}}{k_{B} T}\right)}
\end{aligned}
$$

where $k$ is the wave vector, $h$ is the Planck's constant, $F$ the Fermi Energy, $k_{B}$ is the Boltzmann constant, $T$ the temperature, $m_{l}^{*}$ and $m_{t}^{*}$ are the longitudinal and the transverse effective masses at the $\mathrm{X}$ and $\mathrm{L}$ valleys. $E_{C}^{\mathrm{X}}$ and $E_{C}^{\mathrm{L}}$ are the electron energy at the $\mathrm{X}$ and $\mathrm{L}$ valley, respectively. $E_{k}$ is the kinetic energy. $E_{h h}$ and $E_{l h}$ are the heavy hole and light hole energy, respectively. $E_{D}$ is the ionization energy of donors and $E_{A}$ is the ionization the energy of acceptors. Considering the influence of lattice strain and using the Kane's model (Kane 1957), we get:

$$
\begin{aligned}
& E_{l h}=E_{V, a v}+\Delta E_{V, a v}+\Delta E_{l h}-e \Psi+\frac{E_{g}}{2}\left[1-\sqrt{1+\frac{8 k^{2} P^{2}}{3 E_{g}^{2}}}\right]+\frac{\hbar^{2} k^{2}}{2 m_{0}} \\
& E_{C}^{\Gamma}=E_{V, a v}+\Delta E_{V, a v}+\Delta E_{C}^{\Gamma}-e \Psi+\frac{E_{g}}{2}\left[1+\sqrt{1+\frac{8 k^{2} P^{2}}{3 E_{g}^{2}}}\right]+\frac{\hbar^{2} k^{2}}{2 m_{0}}
\end{aligned}
$$

where $P$ is the momentum matrix element and $m_{0}$ is the rest electron mass.

Standard expressions for energy in the remaining bands after considering the influence of deformations, are expressed as follows:

$$
\begin{gathered}
E_{h h}=E_{V, a v}+\Delta E_{V, a v}+\Delta E_{h h}-e \Psi-\frac{\hbar^{2} k^{2}}{2 m_{h h}} \\
E_{C}^{X}=E_{V, a v}+\Delta E_{V, a v}+\Delta E_{C}^{X}-e \Psi+E_{k} \\
E_{C}^{L}=E_{V, a v}+\Delta E_{V, a v}+\Delta E_{C}^{L}-e \Psi+E_{k}
\end{gathered}
$$

The condition of local electrical neutrality makes it possible to determine the initial values of the electric potential $\Psi_{0}$

$$
N_{D}^{+}(\Psi)-N_{A}^{-}(\Psi)+p_{h h}(\Psi)+p_{l h}(\Psi)-n_{\Gamma}(\Psi)-n_{X}(\Psi)-n_{L}(\Psi)-N_{A}^{-}(\Psi)=0
$$

By solving the Poisson Eq. (14) and considering the shift of the band edges (Eqs. 12 and 13), the spatial distribution of the band structure in the analyzed epitaxial layers was determined. This is shown in Fig. 14a, b (solid lines). In addition, we showed how the band structure distribution would look like if we did not consider the influence of the lattice strain (dashed lines). Choosing the suitable molar composition and the level of doping layers, two-barrier detectors were designed in which the band offset was eliminated. The occurring strain move the edges of the energy band completely differently than in the case of structure B shown in Fig. 14b. Figure 15 shows the distribution of the band structure in the layer B, in which the InAs buffer has a thickness of $10 \mu \mathrm{m}$ and the substrate GaAs has a thickness of $200 \mu \mathrm{m}$. 

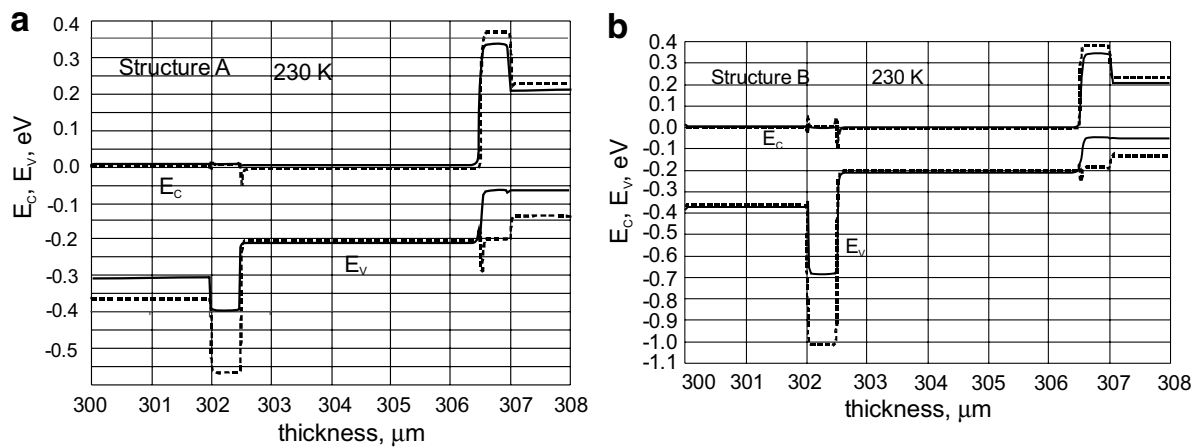

Fig. 14 Spatial distribution of the band structure in thermal equilibrium in structure A (a) and B (b) deposited on $300 \mu \mathrm{m} \mathrm{GaSb}$ and GaAs substrate, respectively. Solid lines show the influence of the lattice strain, dashed lines show the situation when we do not take it into account

Fig. 15 Spatial distribution of the band structure in thermal equilibrium in structure B with $10 \mu \mathrm{m}$ InAs buffer deposited on GaAs substrate with thickness of $200 \mu \mathrm{m}$

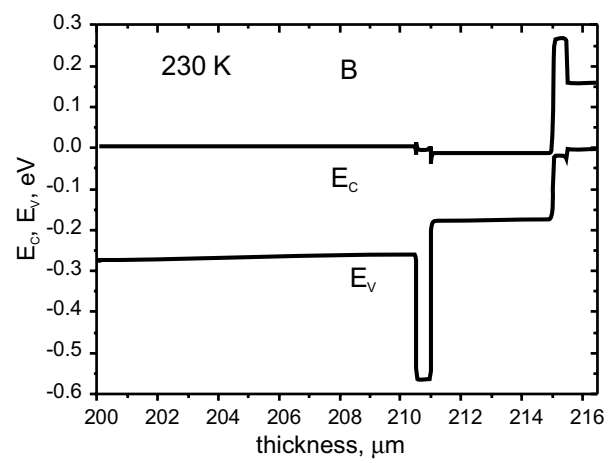

As we can see, by choosing the right thickness of the substrate and the buffer layer, one can affect the size and nature of the lattice strain, and thus affect the spatial distribution of the band structure. Due to large differences in the lattice constant between various layers of the heterostructures from $\mathrm{A}_{\mathrm{III}} \mathrm{B}_{\mathrm{V}}$ compounds, the occurring lattice strains are so significant that when designing heterostructures, it is necessary to include their influence on the position of energy bands.

\section{Conclusions}

The main purpose of the work was to design two-barrier detectors with InAsSb for 3-5, $5 \mu \mathrm{m}$ wavelength range, working at $230 \mathrm{~K}$ temperature. We proposed two solutions of two-barrier detectors in this work. We pointed at the need to conduct enhanced numerical modeling considering the impact of lattice strain on the structure. Applying the heterostructure, that coals the buffer layer and the cap layer from InAs and the absorber from InAsSb, one can build a two-barrier MWIR detector operating at $230 \mathrm{~K}$. As barriers, one can use strong doped thin layers with AlAsSb, which allows to eliminate the band offset. From the minimum elastic energy condition, we determined the density of 
the misfit dislocation in the individual interfaces of the heterostructure. This allows to estimate the partially relaxed lattice stress and strain. As dislocations affect the generation and recombination processes, both the places where they occur, and their density significantly affects the performance parameters of the designed detectors. In this article, apart from pseudomorphic deformations, we considered the bending of heterostructures. In addition, we found a numerical method to determine the density of misfit dislocations at the interfaces of all layers of the heterostructures. We consider this to be the most important element of the novelty in our article.

The most important practical achievement is the ability to design infrared detectors taking into account the influence of the lattice strain and misfit dislocations on their parameters.

Acknowledgements The work has been undertaken under the financial support of the Polish National Science Centre as research Project No DEC-2016/23/B/ST7/03958.

Open Access This article is distributed under the terms of the Creative Commons Attribution 4.0 International License (http://creativecommons.org/licenses/by/4.0/), which permits unrestricted use, distribution, and reproduction in any medium, provided you give appropriate credit to the original author(s) and the source, provide a link to the Creative Commons license, and indicate if changes were made.

\section{References}

Adachi, S.: Properties of IV, III-V and II-VI Semiconductors, p. 218. Wiley, Hoboken (2005)

Ashley, T., Elliott, C.T.: Nonequilibrium devices for infra-red detection. Electron. Lett. 21, 451-452 (1985). https://doi.org/10.1049/el:19850321

Blakemore, J.S.: Semiconductor Statistics, pp. 3-107. Pergamon Press, Oxford (1962)

Capper, P., Garland, J.W.: Mercury Cadmium Telluride, Growth, Properties and Applications, p. 78. Wiley, Hoboken (2011)

Gomółka, E., Kopytko, M., Michalczewski, K., Kubiszyn, Ł., Kębłowski, A., Gawron, W., Martyniuk, P., Piotrowski, J., Rutkowski, J., Rogalski, A.: Electrical and optical performance of mid-wavelength infrared In: AsSb heterostructure detectors. Proc. SPIE, p. 10433 (2017). https://doi.org/10.1117/12.22796 04

Gosling, T.J., Bullough, R., Jain, S.C., Willis, J.R.: Misfit dislocation distributions in capped (buried) strained semiconductor layers. J. Appl. Phys. (1993). https://doi.org/10.1063/1.353445

Hamamatsu (2019) Product catalog https://www.hamamatsu.com/

Jain, S.C., Gosling, T.J., Willis, J.R., Totterdell, D.H.J., Bullough, R.: A new study of critical layer thickness, stability and strain relaxation in pseudomorphic gexsi1-x strained epilayers. Philos. Mag. A 65, 1151-1167 (1992). https://doi.org/10.1080/01418619208201502

Jain, U., Jain, S.C., Nijs, J., Willis, J.R., Bullough, R., Mertens, R.P., Van Overstraeten, R.: Calculation of critical-layer-thickness and strain relaxation in $\mathrm{GexSi}_{1-\mathrm{x}}$ strained layers with interacting 60 and $90^{\circ}$ dislocations. Solid State Electron. 36, 331-337 (1993). https://doi.org/10.1016/0038-1101(93)90084-4

Jain, S.C., Willander, M., Pinardi, K., Maes, H.E.: A review of recent work on stresses and strains in semiconductor heterostructures. Phys. Scr. T69, 65-72 (1997). https://doi.org/10.1088/0031-8949/1997/ T69/009

Jóźwikowska, A.: Numerical solution of the nonlinear Poisson equation for semiconductor devices by application of a diffusion-equation finite difference scheme. J. Appl. Phys. 104, 063715 (2008). https://doi. org/10.1063/1.2982275

Jóźwikowska, A., Jóźwikowski, K., Ciupa, R., Suligowski, M.: Estimation of influence of lattice strain, bending and doping on the width of energy gap in InAsSb heterostructures. Infrared Phys. Technol. 99, 2092-303 (2019). https://doi.org/10.1016/j.infrared.2019.04.020

Jóźwikowski, K., Jóźwikowska, A.: Enhanced numerical modeling of $\mathrm{HgCdTe}$ long wavelength infrared radiation operation temperature non-equilibrium $\mathrm{P}+\nu(\pi) \mathrm{N}+$ photodiodes. J. Electron. Mater. (2019). https://doi.org/10.1007/s11664-019-07264-w

Kane, E.O.: Band structure of indium antimonide. J. Phys. Chem. Solids 1, 249-261 (1957). https://doi. org/10.1016/0022-3697(57)90013-6 
Kim, J.D., Razeghi, M.: Investigation of InAsSb infrared photodetectors for near room temperature operation. Opto-Electron. Rev. 6(3), 217-230 (1998)

Klipstein, P., Klin, O., Grossman, S., Snapi, N., Lukomsky, I., Yassen, M.D., Aronov, D., Berkowitz, E., Glozman, A., Magen, O., Shtrichman, I., Frenkel, R., Weiss, E.: High operating temperature XBnInAsSb bariode detectors. In: Proc. SPIE, p. 8268 (2012). https://doi.org/10.1117/12.910174

Look, D.C.: Electrical Characterization of GaAs Materials and Devicees, pp. 107-132. Wiley, New York (1989)

Plis, E., Rodriguez, J.B., Balakrishnan, G., Sharma, Y.D., Kim, H.S., Rotter, T., Krishna, S.: Mid-infrared InAs/GaSb strained layer superlattice detectors with $\mathrm{nBn}$ design grown on a GaAs substrate. Semicond. Sci. Technol. 25(8), 085010 (2010). https://doi.org/10.1088/0268-1242/25/8/085010

Pinardi, K., Maes, H., Jain, S., Jain, U., Willander, M.: Structure of II-VI lattice mismatched epilayers used for blue-green lasers for underwater communication. Def Sci J 48, 31-43 (2013). https://doi. org/10.14429/dsj. 48.3865

Singh, J.: Physics of semiconductors and their heterostructures, pp. 1-26. McGraw-Hill Inc., New York (1993)

Smith, D.L., Mailhiot, C.: Proposal for strained type II superlattice infrared detectors. J. Appl. Phys. 32, 2545-2548 (1987). https://doi.org/10.1063/1.339468

Soibel, A., Hill, C.J., Keo, S.A., Hoglund, L., Rosenberg, R., Kowalczyk, R., Khoshakhlagh, A., Fisher, A., Ting, D.Z., Gunapala, S.D.: Room temperature performance of mid-wavelength infrared InAsSb $\mathrm{nBn}$ detectors. Infrared Phys. Technol. 70, 121-124 (2015). https://doi.org/10.1016/j.infrared.2014.09.030

Soibel, A., Ting, D.Z., Hill, C.J., Fisher, A.M., Hoglund, L., Keo, S.A., Gunapala, S.D.: Mid-wavelength infrared InAsSb/InSb nBn detector with extended cut-off wavelength. Appl. Phys. Lett. 109, 103505 (2016). https://doi.org/10.1063/1.4962271

Ting, D.Z., Soibel, A., Hoglund, L., Hill, C.J., Keo, S.A., Fisher, A., Gunapala, S.D.: High-temperature characteristics of an InAsSb/AlAsSb n + Bn detector. J. Electron. Mater. 45, 4680-4685 (2016). https:// doi.org/10.1007/s11664-016-4633-Z

Van de Walle, C.G.: Band lineups and deformation potentials in the model-solid theory. Phys. Rev. B 39(3), 1871-1883 (1989). https://doi.org/10.1103/PhysRevB.39.1871

Vigo System SA (2019) Product catalog https://vigo.com.pl/

Vurgaftman, I., Mayer, J.R., Ram-Mohan, L.R.: Band parameters for III-V compound semiconductors and their alloys. J. Appl. Phys. 89, 5815-5875 (2001). https://doi.org/10.1063/1.1368156

White, A.M.: Generation-recombination processes and Auger suppression in small-bandgap detectors. J. Cryst. Growth 84, 840-848 (1988). https://doi.org/10.1016/0022-0248(90)90813-Z

Xie, C., Pusino, V., Khalid, A., Craig, A.P., Marshall, A., Cumming, D.R.S.: Monolithically integrated InAsSb-based $\mathrm{nBnBn}$ heterostructure on GaAs for infrared detection. IEEE J. Sel. Top. Quantum Electron. 24, 1-6 (2018). https://doi.org/10.1109/JSTQE.2018.2828101

Publisher's Note Springer Nature remains neutral with regard to jurisdictional claims in published maps and institutional affiliations. 